\title{
High Resolution Photoacoustic System Based on Acoustic Lens with Negative Refractive Index
}

\author{
Jianning Han ${ }^{1}$, Quan Zhang ${ }^{1}$, Peng Yang ${ }^{1}$ and Yifan Gong ${ }^{1}$ \\ ${ }^{1}$ School of Information and Communication Engineering, North University of \\ China \\ Tai Yuan, Shan Xi, 030051 \\ ${ }^{1}$ Tel.: 13994203384 \\ ${ }^{1}$ E-mail:yp7458@sina.cn
}

\begin{abstract}
As a new nondestructive biophotonic technology, taking the ultrasonic wave as the media, opto-acoustic tomography is a kind of imaging method based on the differences of optical absorption within the biological organization. With an analysis on the loss of the high-frequency information containing image details resulted from the decay of the evanescent wave in the traditional acoustic lens in the current opto-acoustic tomography system, a study is conducted in this paper by using the lens with negative refractive index to make the evanescent wave participate in the imaging process, and break through the diffraction limit of the traditional acoustic lens, so as to greatly improve the resolution of image. Moreover, according to the characteristics of ultrasonic wave produced in opto-acoustic effect, the relevant theory of acoustic information system and three-dimensional acoustic imaging technology are supplemented and developed in the study. In addition, a set of comparative experiment is done on the imaging effect between the traditional lens and the lens designed in this paper, and the experiment shows that the imaging effect of opto-acoustic tomography within the biological organization got by the designed lens greatly beyond that of the traditional lens.
\end{abstract}

Keywords: Opto-acoustic Tomography, Acoustic Lens, Negative Refraction, Resolution

\section{Introduction}

With the increasing development of the technology of computer, material and industry, a considerable progress has been made on the opto-acoustic tomography in medical ultrasound imaging. Especially, in the recent years, an earthshaking change is made on the new methods, techniques and materials in the opto-acoustic tomography, which also paves a way for the commercial applications of the opto-acoustic tomography. Using the outward radiant ultrasonic wave resulted from the thermal expansion produced by the laser exposed on the biological organization, opto-acoustic tomography is an imaging technology to obtain the image of the organization, and its important value of studying and popularizing is shown in the diagnosis conducted by Wang Lihong's group at the University of Washington on the early cancer [1].

Moreover, there have been some typical experimental applications of opto-acoustic tomography technology. For examples, Ronald E. Kumon, et al., working in the Department of Biomedical Engineering, Ann Arbor Campus, University of Michigan, used the opto-acoustic tomography technology to make a frequency-domain analysis on the prostate cancer tumor in the body of white rat, and achieved some good findings [2]; Xueding Wang, et al., working in the Department of Radiological Sciences, Ann Arbor Campus, University of Michigan, used the commercial imaging equipment combining with the detector developed by themselves to make image on a double of human hair and the treelike vascular of rabbit's ear, and acquired some quite clear images [3]; in China, 
Hui Wang, et al., working in the Key Laboratory of Laser Life Science, Ministry of Education, South China Normal University, used Fresnel zone plate ultrasonic detector to make images, and realized opto-acoustic tomography [4]. Except for the examples above, some correlative studies on the technology of opto-acoustic tomography were conducted in other research institutions. On the whole, they mainly conducted their researches from two aspects: one is to use new materials, or make up the sensors array, to ameliorate the receiving sensor; the other is to use the new filtering and rebuilding algorithm of image to make a post-treatment on the images. However, all of the studies don't solve the problems caused by the technology of opto-acoustic tomography described above, in specific, the spatial resolution of imaging is not high, and some tiny organizations still can' t be distinguished. In addition, when the imaging experiment is conducted on the living body, the quality of imaging is greatly impacted by the complex noise caused by the technology.

Since Veselago of the former Soviet Union proposed the concept of left-handed material [5] (negative refraction material) in 1968, the enthusiasm of scientists in the study of negative refraction materials has not been reduced. Especially in 2000, J. B. Pendry published an article named Negative Refraction Makes a Perfect Lens [6] on the Phys. Rev. Lett, which induced a series of discussion on the topic of negative refraction materials.

Negative refraction acoustic lens is just the one of lens compounded of different negative refraction mediums, and these mediums are compounded according to a certain rule, which shares the characteristic of negative refraction [7]. Moreover, this kind of lens has some merits, such as, focusing, band gap, direction propagation and etc. In addition, the characteristics of this kind of lens, such as, focusing, filtering and directional control on acoustic wave, are very suitable for the resolution of the inherent disadvantages of the technology of opto-acoustic tomography in opto-acoustic tomography system.

From the literature, it can be seen that, up to now, many research institutions use acoustic lens to realize opto-acoustic tomography, but few of the studies are conducted on the application of negative refraction acoustic lens in opto-acoustic tomography system [8-10]. In this paper, with an analysis on the loss of the high-frequency information containing image details resulted from the decay of the evanescent wave in the traditional acoustic lens in the current opto-acoustic tomography system, a study is conducted by using the lens with negative refractive index to make the evanescent wave participate in the imaging process, and break through the diffraction limit of the traditional acoustic lens, so as to greatly improve the resolution of image. At the same time, the relevant theory of acoustic information system and three-dimensional acoustic imaging technology are supplemented and developed in the study.

\section{Establishment of the Negative Refraction Acoustic Lens Model}

As shown in the Figure 1, the nanosecond pulse laser irradiate is generally applied into the current opto-acoustic tomography system to make an exposure on the detection area. When the detection is conducted, the detection area is expanded because of the heat of the laser. Due to the difference between the indexes of refraction of the diseased and normal organization, the coefficients of acousto-optic transition of the diseased and normal organization are different, which directly results in the difference between the thermal expansions of the mediums. Thus, different acoustic signals are produced in the two organizations. In addition, the signals can be received in the form of ultrasonic wave, and the distribution of the optical absorption inside the target can be shown in the image after reconstruction. Therefore, it can be concluded that the key to opto-acoustic tomography is how to detect the ultrasonic signals. For the convenience of study, in this paper, the point radiating acoustic wave under the exposure of laser is simplified as point source in acoustic research, and the core of the study is how to detect the imaging information of the point source with high quality. Currently, some researchers have initiated the study on 
ordinary acoustic lens. To be more specific, set on the left side of the lens, the point source radiates waves under the exposure of laser, and the waves are gathered at the focal point on the right side of the lens through the transmission of acoustic lens, as shown in the Figure 2. However, because of the ordinary acoustic lens in the core of the lens for imaging, the decay of the evanescent wave in the ordinary acoustic lens results in the loss of the high-frequency information containing image details. In addition, the effect of the imaging is not ideal, as shown in the Figure 3.

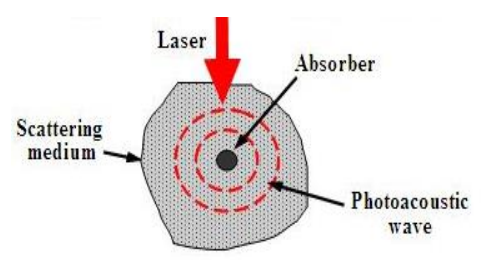

Figure 1. Thermal Expansion of Biological organization

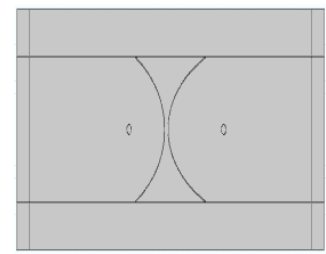

Figure 2. Diagrammatic Sketch for the Imaging of Ordinary Lens

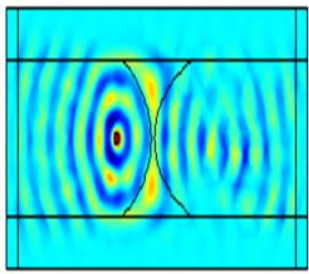

Figure 3. Diagrammatic Sketch for the Imaging of Ordinary Lens

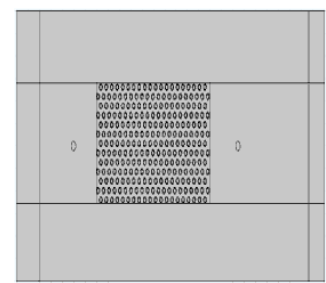

Figure 4. Diagrammatic Sketch for the Imaging of Negative Refraction Lens

In this paper, for the first time, the application of the negative refraction lens into the opto-acoustic tomography system is put forward. Being set on the left side of the lens, the point source radiates waves under the exposure of laser, and the waves are gathered on the right side of the lens through the transmission of acoustic lens [11]. Then, the imaging is conducted by using the advantages of negative refraction lens, such as, focusing, filtering and directional control on acoustic wave, as shown in the Figure 4. From this Figure, it can be seen that the participation of evanescent wave in the imaging process solves the problems of the decay of evanescent wave and the loss of high-frequency information containing image details in traditional acoustic lens, greatly improves the resolution of image, and supplements the inherent disadvantages of the technology of ordinary opto-acoustic tomography. 


\section{Theoretical Analysis on Negative Refraction Imaging}

As a kind of ultrasonic wave excited by light, opto-acoustic signal substantially has all the characteristics of the wave. Therefore, provided that, (1) a pulsed laser with uniform distribution (The pulse width is magnitude) is used to make exposure on the biological organization, and the dimension of opto-acoustic signal generated by each point in the biological organization is proportional to the light absorption coefficient of the point in biological organization; (2) each opto-acoustic signal generated by the points in the biological organization is a point source with amplitude decayed by $1 / \mathrm{r}$, and the function of the wave generated by the point source is $\varphi=U e^{j w t}$, moreover, in this function, $U$ represents the complex amplitude of sound field. [12]When the acoustic wave is emitted onto the plane $(\mathrm{x}, \mathrm{y})$, there is a component for the wave propagated along the direction Z. According to the scalar wave equation of Helmholtz $\nabla^{2} U+k^{2} U=0$, when the distance of propagation along the direction $\mathrm{z}$ of the wave is enough short, the solution to the wave equation can be expressed in the form of:

$$
A\left(k_{x}, k_{y}, z_{0}\right)=A\left(k_{x}, k_{y}, 0\right) e^{j k_{z} z_{0}}
$$

Hereinto, $A\left(k_{x}, k_{y}, z_{0}\right)$ is the spatial spectrum distribution of the plane.

Considering that the wave is propagated in the three-dimensional space, according to the relationship among $\mathrm{k}_{\mathrm{x}}, \mathrm{k}_{\mathrm{y}}$ and $\mathrm{k}_{\mathrm{z}}$, the expression $k_{x}, k_{y}, k_{z}$ can be used to express the wave vector of free space. Therefore, the equation $\Delta=2 \pi / k_{\max }=2 \pi c / \omega=\lambda$ can be deduced, hereinto, $k_{0}=2 \pi / \lambda$

In the same time, considering that the index of refraction of the lens is positive or negative, the equation $k_{z}= \pm \sqrt{k_{0}{ }^{2}-\left(k_{x}{ }^{2}+k_{y}{ }^{2}\right)}= \pm \sqrt{n^{2} \omega^{2} / c^{2}-\left(k_{x}{ }^{2}+k_{y}{ }^{2}\right)}$ can be deduced, hereinto, $k_{z}$ is positive in the positive refraction lens and negative in the negative refraction lens.

$$
\text { when }{k_{x}}^{2}+k_{y}{ }^{2}>{k_{0}}^{2} \text {, so } k_{z}=j \sqrt{\left(k_{x}{ }^{2}+k_{y}{ }^{2}\right)-k_{0}{ }^{2}}=j \zeta \quad \text { (pure imaginary number) }
$$

At present, the $k_{z}$ is a pure imaginary number, so $A\left(k_{x}, k_{y}, z_{0}\right)=A\left(k_{x}, k_{y}, 0\right) e^{-\zeta z_{0}}$, it leads the resolution limit of the lens $\Delta=2 \pi / k_{\max }=2 \pi c / \omega=\lambda$, obviously, When an acoustic wave propagate in the positive index lenses, wave vector $k_{z}$ is change into a imaginary number. The resolution limit is a wavelength, Although the Radiation wave field phase of body surface can carry more fine structure information remained unchanged in the direction of propagation, the amplitude will have an exponential attenuation. This part carries high surface fine structure information of evanescent attenuation called evanescent wave. It can be observed that the attenuation of positive refractive index lens in Figure 5, so the information of fine structure carried can't go to the far field, and they are limited in the near-field area close to the lens. That Lead to a low resolution of ordinary lens, it can't satisfy with the need of opto-acoustic tomography. 


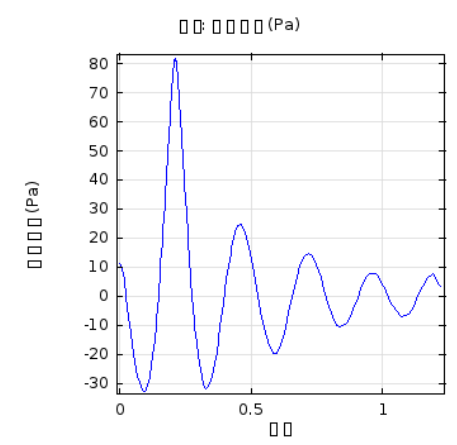

Figure 5. The Evanescent Wave of Common Lens

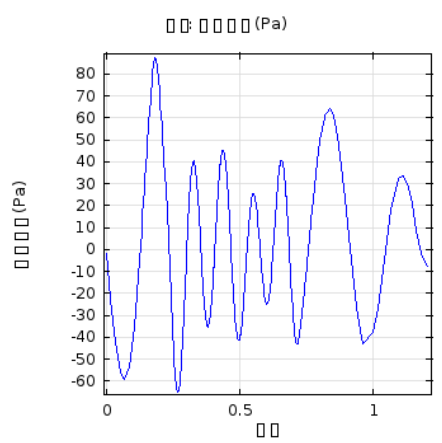

Figure 6. The Enhanced Evanescent Wave of Negative Refraction Lens

According to the acoustic lens medium material ideas continue to analysis, if the transmission medium in the opposite direction, when $\mathrm{n}<0$, the $k_{x}{ }^{2}+k_{y}{ }^{2}>k_{0}{ }^{2}$, so $k_{z}=-j \sqrt{\left(k_{x}{ }^{2}+k_{y}{ }^{2}\right)-k_{0}{ }^{2}}=-j \zeta$ (pure imaginary number), the (1) can express as $A\left(k_{x}, k_{y}, z_{0}\right)=A\left(k_{x}, k_{y}, 0\right) e^{-j^{*} j \xi z_{0}}=A\left(k_{x}, k_{y}, 0\right) e^{\xi z_{0}}$. Although the propagation constants is change into the imaginary, but the amplitude will be changing as $e^{\xi z}$ exponential form, acoustic amplitude along the $\mathrm{Z}$ direction is not decay as exponential form, therefore, the evanescent wave of carrying surface fine structure information can retain well, it can't decay just like in the positive refractive index medium. We can use that transmission medium has a negative refractive index to break the diffraction limit, considering to make the evanescent wave involved in the imaging. Figure 6 shows the evanescent waves in the flat lens is amplify as exponentially, more and more high frequency component is able to transport, it can effectively make up for the exponential decay of evanescent wave in the water. So that it can be successfully transferred to the image plane involved in imaging. Therefore, this design of essay using the negative refraction lens in the opto-acoustic tomography, Figure 7 simulate the negative refractive index lens imaging effect. From the result of simulation, we can see after the original point source through negative refraction lens, it converge into a clear image point on the right side lens. Figure 8 is the simplified model for the opto-acoustic tomography, the model analysis is consistent with Figure 4. It Show that the model is scientific. From the point in the waveform as shown in Figure 9 can be seen that the resolution of the lens reaches 0.3 wavelengths, so that the lens design is a better design of a high resolution opto-acoustic tomography theoretically. 


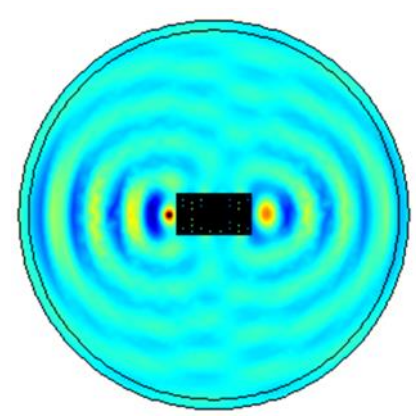

Figure 7. Simulation Effect of the Negative Refractive Imaging

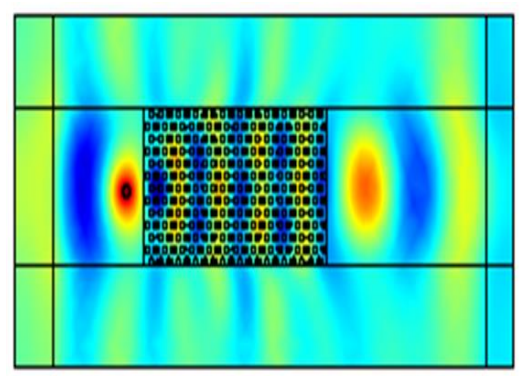

Figure 8. Amplified Analog Effect for the Negative Refractive Imaging

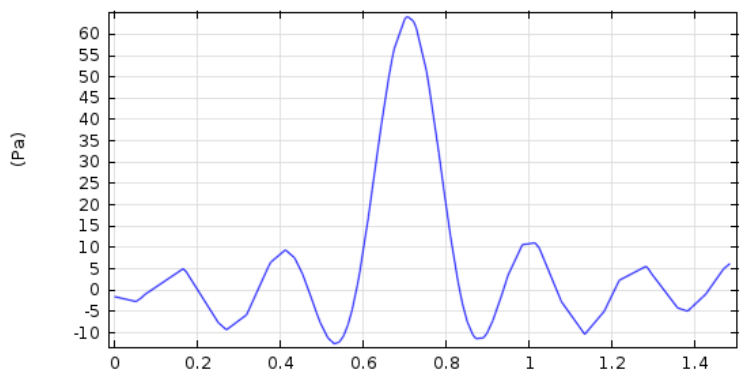

Figure 9. Sound Pressure Distribution of the Focus Image Point

\section{Experimental Method and Result Analysis}

\subsection{Experimental Method and Process}

Based on the above model of the lens, we build the acoustic lens opto-acoustic experiment system as shown in Figure 10. In order to distinguish different experiment effect of the lens, we choose two kind of refraction index lens making experiment. We use four lasers YAG by the American company (Quanta-Ray PIV, Spectrum Physics). Pulse repetition frequency of $25 \mathrm{KHZ}$, pulse width of $10 \mathrm{~ns}$, wavelength of $1064 \mathrm{~nm}$, The incident light spot diameter of $1 \mathrm{~mm}$. the sample used in the experiment is an artificial production with test panel of calibration, Panel placed on a human hair, put it at different distances for display the maximum resolution of imaging system can be distinguish. The matrix surrounding sample box $\mathrm{f}$ is liquid oil, putting the acoustic lens on the sample box; the acoustic sensor array is placed on the focus plane of the lens. For distinguish contrast effects, using two acoustic lens, one is ordinary acoustic lens composed of resin materials, another one is negative refraction acoustic lens composed of coat rubber layer by shot embedded in epoxy resin. Receiving array is the $16 \times 16$ micro-nano ultrasonic sensor, then connect to the data acquisition circuit after $16 \times 16$ channels data bus is connected. 


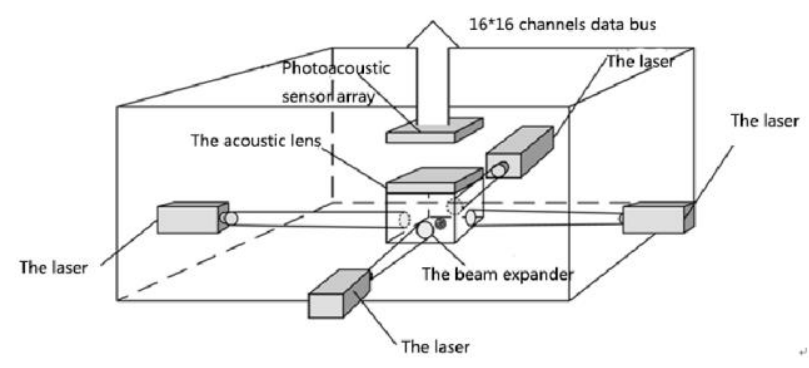

\section{Figure 10. Experimental System of Acoustic Lens Opto-acoustic Tomography}

\section{Experimentation:}

1) Put the sample in the test bench of frosted glass vessels.

2) Open the laser, through the beam expander and frosted glass beam expander, make the laser on the samples as uniform.

3) The samples by laser irradiation will heat up and expansion, then it will irradiate the acoustic waves. These acoustic waves through the acoustic lens (general lens and negative refraction lens) focus in focal plane of opto-acoustic sensor array composed of 256 element of $16^{*} 16$.

4) Opto-acoustic sensors make the acoustic signals of sample change into electrical signals, And get the analog signal through the filter amplifier signal conditioning, then the analog signal is converted to a digital signal through the data acquisition card. According to the rules of the lens, we get the two-dimensional image model of the hair.

5) Each opto-acoustic sensor receives the characteristics of ultrasonic signal in the time and amplitude domain, transmitted into RAM, and combined with spatial information of acoustic lens imaging model, we can get the final result of opto-acoustic tomography data.

6) The imaging data after processing can get the opto-acoustic tomography results eventually.

\subsection{The Experimental Result and Analysis}

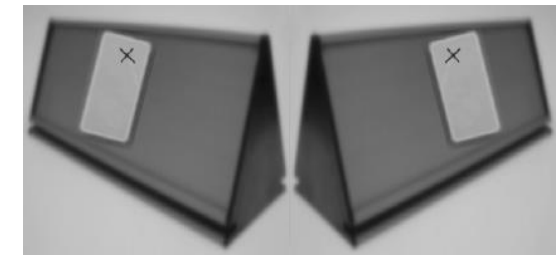

Figure 11. Experimental Sample

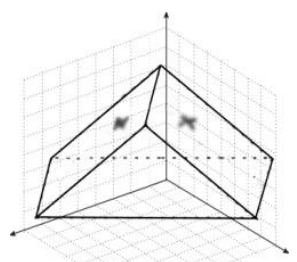

Figure 12. Ordinary Acoustic Lens Imaging 


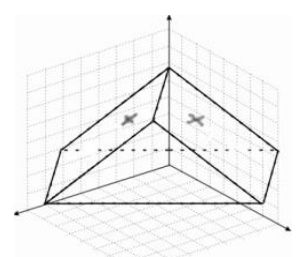

\section{Figure 13. Negative Refraction Lens Imaging}

The experimental sample in Figure 11 shows that cross-shaped hair on the top. The hair by laser irradiate ultrasonic, its imaging effect through ordinary lens as show in Figure 12, the image is fuzzy, even can't identify the cross-shaped of the hair. In order to distinguish imaging effect, using negative refraction lens. The hair by laser irradiate ultrasonic, its imaging effect through negative refraction lens as show in Figure 13, the image is very clear, the cross-shaped of the hair is clearly visible.

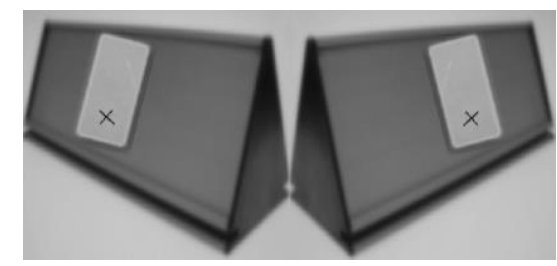

Figure 14. Experimental Sample

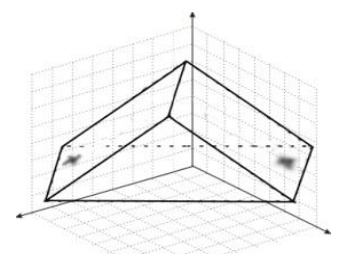

Figure 15. Ordinary Acoustic Lens Imaging

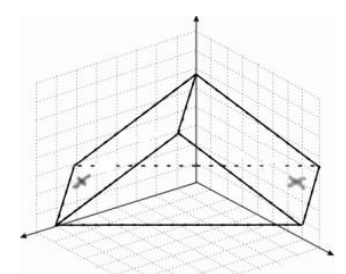

Figure 16. Negative Refraction Lens Imaging

The experimental sample in Figure 14 shows that cross-shaped hair on the bottom. The hair by laser irradiate ultrasonic, its imaging effect through ordinary lens as show in Figure 15, the image is fuzzy. The hair by laser irradiate ultrasonic, its imaging effect through negative refraction lens as show in Figure 16, the image is very clear. On the basis of above experiment images combined with lens imaging knowledge, we can know because of the limitation of the ordinary lens imaging system resolution limit, there is a virtual image of centering on the absorber and spread outward, It around the concentrated absorber (Hair intersection), it Make up the fuzzy intersection obviously. However, the image around the concentrated absorber (Hair intersection) is very clear in negative refraction lens, it is easy to identify, and the image is consistent with the original sample. The result is very well explain that negative refraction lens is superior to the traditional lens in imaging resolution. 


\section{Conclusion}

First of all, the essay obtains a result that the opto-acoustic tomography of negative refraction lens can enhance the evanescent wave from theoretical analysis and physical model. It makes the evanescent wave take part into the imaging, which increases the resolution ratio of lens imaging. Analysis the cross-shaped hair through lens imaging model, we gain the best imaging result of this experiment system by the imaging comparison of a lot of experiment sample. The result shows the imaging effect of negative refraction lens is better than the normal acoustic lens. The result plays an important role of enhance opto-acoustic tomography in the field of medical research and clinical examination. As for the weekness is the less of the unit of sensor array, limit the possibility of higher resolution ratio. In addition, we use normal image processing in later image processing. If we can use more advanced and fit image processing technology, we will get a better image resolution.

\section{Acknowledgements}

This project was sponsored in part by The National NaturalScience Foundation of Chinese NO.61302159.

\section{References}

[1] M. Pramanik, G. Ku, C. Li and L. V. Wang, "Design and evaluation of a novel breast cancer detection system combining both thermoacoustic (TA) and photoacoustic (PA) tomography", Medical physics, vol. 35, no. 6, (2008), pp. 2218-2223.

[2] R. E. Kumon, C. X. Deng and X. Wang, "Frequency-domain analysis of opto-acoustic imaging data from prostate adenocarcinoma tumors in a murine model", Ultrasound in medicine \& biology, vol. 37, no. 5, (2011), pp. 834-839.

[3] X. Wang, J. B. Fowlkes, J. M. Cannata, C. Hu and P. L. Carson, "Opto-acoustic imaging with a commercial ultrasound system and a custom probe", Ultrasound in medicine \& biology, vol. 37, no. 3, (2011), pp. 484-492.

[4] H. Wang, D. Xing and L. Xiang, "Photoacoustic imaging using an ultrasonic Fresnel zone plate transducer", Journal of Physics D: Applied Physics, vol. 41, no. 9, (2008), pp. 095111.

[5] V. G. Veselago, "THE ELECTRODYNAMICS OF SUBSTANCES WITH SIMULTANEOUSLY NEGATIVE VALUES OF IMG align= ABSMIDDLE alt= $\epsilon$ eps/IMG AND $\mu$ ”, Physics-Uspekhi, vol. 10, no. 4, (1968), pp. 509-514.

[6] J. B. Pendry, "Negative refraction makes a perfect lens", Physical review letters, vol. 85, no. 18, (2000), pp. 3966.

[7] C. Bao and J. M. Castresana, "Chiral waves in a metamaterial medium", In SPIE Photonics Europe, International Society for Optics and Photonics, (2010) April, pp. 77111Y-77111Y.

[8] K. Maslov, H. F. Zhang, S. Hu and L. V. Wang, "Optical-resolution opto-acoustic microscopy for $<\mathrm{i}>$ in vivo</i> imaging of single capillaries", Optics letters, vol. 33, no. 9, (2008), pp. 929-931.

[9] L. V. Wang and S. Hu, "Photoacoustic tomography: in vivo imaging from organelles to organs", Science, vol. 335, no. 6075, (2012), pp. 1458-1462.

[10] L. V. Wang and S. Hu, "Opto-acoustic tomography: in vivo imaging from organelles to organs", Science, vol. 335, no. 6075, (2012), pp. 1458-1462.

[11] Z. Liu, X. Zhang, Y. Mao, Y. Y. Zhu, Z. Yang, C. T. Chan and P. Sheng, "Locally resonant sonic materials", Science, vol. 289, no. 5485, (2000), pp. 1734-1736.

[12] N. A. Gumerov and R. Duraiswami, "Fast multipole methods for the Helmholtz equation in three dimensions", Elsevier, (2005).

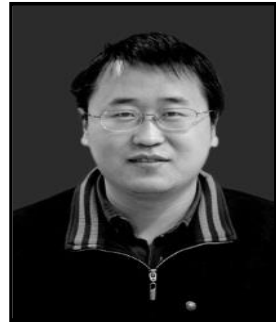

\section{Authors}

Jianning Han, School of Information and Communication Engineering, North University of China, Taiyuan Shanxi China 
International Journal of Signal Processing, Image Processing and Pattern Recognition Vol. 8, No. 4 (2015) 